\title{
La complejidad y la transdisciplina como sistemas teóricos en el hacer de la educación superior*
}

\author{
J. Loreto Salvador Benítez ${ }^{1}$ \\ (D) http://orcid.org/0000-0003-3438-9539 \\ Sergio González López ${ }^{2}$ \\ (iD http://orcid.org/0000-0003-4547-4210 \\ Universidad Autónoma del Estado de México
}

DOI: http://dx.doi.org/10.17081/eduhum.19.33.2646

Recibido: 20 de junio de 2016

Aceptado: 18 de marzo de 2017

\section{Complexity and transdiciplinarity as theoretical systems in higher education}

Palabras clave:

Complejidad, Educación,

Sustentabilidad, Transdisciplina.

Key words:

Complexity, Education,

Sustainability, Transdiscipline.

\begin{abstract}
Resumen
Se presenta la ciencia moderna tradicional antepuesta a las ideas de la complejidad y transdisciplina como maneras de abordar y generar conocimiento desde la perspectiva de los sistemas complejos. Se proponen opciones metodológicas, epistémicas y teóricas en contraoposición al paradigma actual de la ciencia moderna, objetiva y cuantitativa. Esto se lleva al terreno de la educación superior, donde tienen lugar acciones y análisis de estos contenidos. Asimismo se plantean ejemplos en la propia educación terciaria y en la noción actual de la sustentabilidad, de sumo compleja, cuyo punto de interés vuelve a la naturaleza y la vida.
\end{abstract}

\begin{abstract}
Modern science is characterized for its contrast with the ideas of complexity and transdiscipline, as ways to approach and generate knowledge from the perspective of complex systems. The current paradigm of modern, objective and quantitative science is proposed as a methodological, epistemic and theoretical option. This takes us to the field of higher education where the actions and analysis of these concepts take place. Likewise, examples are proposed in tertiary education itself and in the current notion of sustainability, complex as it is whose main point of interest take us back to nature and life.
\end{abstract}

\section{@) $(\Theta \Theta$}

Referencia de este artículo (APA): Salvador, J.L. \& González, S. (2017). La complejidad y la transdisciplina como sistemas teóricos en el hacer de la educación superior. En Revista Educación y Humanismo, 19(33), 305-319. http://dx.doi.org/10.17081/ eduhum.19.33.2646

* Artículo derivado de la línea de investigación de generación y aplicación del conocimiento.

1. Profesor investigador adscrito al Instituto de Estudios sobre la Universidad. Cuerpo Académico: Estudios sobre la Universidad. sloreto79@yahoo.com

2. Doctor en urbanismo. Profesor de la Universidad Autonóma de México. sergiogonlop2@yahoo.mx 


\section{Introducción}

Este es un trabajo conceptual-reflexivo que analiza dos conceptos actuales en la teoría y la práctica científica, los cuales se tocan metodológicamente en la acción docente y de investigación en los procesos de la Universidad. Uno de estos conceptos es la complejidad, que se expresa en hipótesis y teorías de diversas disciplinas; y el otro, la transdisciplina, presente en la práctica y abordaje de objetos de estudio, al reconocer la insuficiencia en la comprensión-explicación de un hecho o fenómeno, en sí mismos multidimensionales, desde una sola óptica disciplinar. Se trata de una discusión aún en curso en las ciencias, que se replica en las universidades desde y en la formación profesional disciplinar.

$\mathrm{Y}$ es que ahora es indiscutible que frente a la histórica tradición de la ciencia moderna, experimental y cuantitativa, han surgido otras argumentaciones que van más allá de su método de comprensión y praxis, y cuyos aportes al conocimiento son incuestionables. Tal, como ya se dijo, es la idea de "complejidad" que se antepone, precisamente, a la disyunción y reducción de características de la ciencia moderna. Desde esta perspectiva, en lugar de la parte como objeto de estudio, se observa el sistema o totalidad del proceso o fenómeno, procurando atender la diversidad de vínculos que se desprenden de ellos.

El otro cuerpo teórico en construcción es la transdisciplina, que se vincula estrechamente con la complejidad. Son planteamientos metodológicos y epistémicos que desde distintas ciencias, en mayor o menor medida, han estado interactuando en estudios e investigaciones. Metodológicamente, se expone primero una caracterización de la ciencia moderna con la intención de contrastar la noción de complejidad con la disyunción y reducción, dado que en la complejidad el objeto de estudio e interés sobresale por sus nexos con otras circunstancias, entidades o fenómenos; esto es, que se aspira a superar la perspectiva causística de sujeto-objeto para prestar atención a una totalidad o unidad en tanto sistema.

\section{Problema de investigación}

La academia y la ciencia actuales proceden de la racionalidad moderna que aún impera en la cultura global, institucional y tecnológica que da sentido a acciones y hechos en la vida social humana y en su correlación e impacto con la naturaleza, los ecosistemas regionales e, incluso, la biosfera y la Tierra como unidad y totalidad. Se trata de una racionalidad instrumental que ha mostrado, desde los paradigmas del mecanicismo, la experimentación y la cuantificación (matemática), <<certezas $>>$ respecto a los hechos y fenómenos de la naturaleza, incluidos en esta el hombre y el cosmos. Una gran tradición epistemológica y metodológica ha contribuido así al desarrollo de las disciplinas científicas. Las reglas del Método cartesiano de la Evidencia, el Análisis (dividir todo problema en tantas partes como sea posible; reducción de lo complejo a sus elementos simples); la Síntesis, conducir con orden el pensamiento, de los objetos más simples 
de conocer al saber más complejo; y la Enumeración, aseguran un análisis completo y una síntesis correcta, previniendo el error al controlar los pasos anteriores (Reale \& Antiseri, 2001). Simultáneamente a la racionalidad moderna, fue configurándose una ciencia también moderna a partir de la experimentación, del control del objeto de estudio, y de su medición y explicación como puntas de lanza en la generación de certezas y conocimiento del $<<$ orden $>>$ del mundo, sobre el cual, desde tiempo atrás, había establecido la metáfora de una naturaleza escrita en lenguaje matemático que habría que decodificar. En su texto El Mundo, Descartes plantea una equivalencia entre las leyes de la naturaleza y las eternas verdades, siendo estas -interpreta Flórez (2011) - "las que nos enseñan que Dios ha creado todo $<<$ según número, peso y medida $>>$, que sería el código que opera en la naturaleza y que no es otro que el orden y medida a que se refiere la mathesisuniversalis" (p.9). Ante esta gran tradición en la generación del conocimiento científico, al paso del tiempo, emergieron diversas hipótesis y teorías que ponen en entredicho ese supuesto $<<$ orden $>>$ y las certezas asumidas en los discursos científicos. Comienzan a surgir así otras orientaciones y perspectivas que van de la parte, del objeto de atención y estudio, al conjunto como sistema o totalidad. En este marco, la idea de complejidad, entre otras, se plantea como posibilidad comprensiva en los ámbitos epistémicos, metodológicos y teóricos. Por otra parte, podríase coincidir con Capra (2007) en que uno de los aspectos más radicales del Nuevo paradigma científico es: "la ausencia de cualquier fundamento firme". Y en toda esta controversia académica, científica e intelectual, la Universidad resulta ser el escenario ad hoc, por cuanto en el campus el saber se analiza, comparte, critica, crea y recrea.

\section{Método}

Este trabajo consiste en un análisis teórico documental en el contexto del método hipotético deductivo; se parte de la exposición de enunciados conceptuales con pretensión de valor cognitivo y extensión universal. Se exponen y analizan ideas a partir de la $<<$ complejidad $>>$ que, en tanto discurso innovador, aspira a confrontar y superar la disyunción y reducción de derivados de la modernidad científica aún imperante en muchas disciplinas. El planteamiento teórico deriva en una propuesta de pensamiento, en el caso de Edgar Morin (2003), pero también es posible identificarlo en diversas argumentaciones hipotéticas que esgrimen argumentos para explicar y comprender los hechos y fenómenos sociales y naturales e, incluso, cósmicos. Interesa aquí extender y vincular metódica y epistémicamente la complejidad con los conceptos que, en sí mismos, son de naturaleza compleja, en el entendido de los distintos lazos o vertientes que concurren en ellos, como son los casos de la educación, la sustentabilidad y la transdisciplina. Esto se presenta a manera de ejemplo concreto, y no como una solución o respuesta definitiva alguna, dado que, en el sentido del devenir natural y humano, todo está en constante cambio y evolución, nada es estático. Es posible abordar, comprender y dar sentido a tal constitución de la 
$<<$ realidad $>>$ desde la perspectiva de totalidad, llámese sistémica o fenomenológica.

\section{Idea e interés sobre la complejidad}

Para contextualizar y comprender por oposición la noción de $<<$ complejidad $>>$ habrá que partir de las disciplinas científicas, y particularmente del método cartesiano que propone cuatro reglas: $1^{\mathrm{a}}$ De la Evidencia, $2^{\mathrm{a}}$ del Análisis, $3^{\mathrm{a}}$ Síntesis y $4^{\mathrm{a}}$ Enumeración. Esta última consiste en comprobar constantemente los pasos dados en el análisis y en la síntesis para evitar errores. En opinión de Morin, el primer pensador que hace surgir de manera radical la dualidad que marcaría al Occidente moderno es Descartes, quien postula un universo objetivo de la res extensa alternadamente con el cogito subjetivo como principio primero de la realidad.

La pretensión de "hechos objetivos", al margen de todo juicio de valor, de toda "deformación subjetiva" en aras del método experimental y sus técnicas de verificación, han detonado el crecimiento de la ciencia moderna. Hasta el momento lo científico ha consistido en deshacerse de la imprecisión, la contradicción y ambigüedad; sin embargo, es necesario reconocer la imprecisión en fenómenos y conceptos, así como la creatividad y la libertad entre muchas otras complejidades: biológica, micro y macrofísica, psíquica, social.

El paradigma de la simplicidad ordena el universo y "persigue al desorden"; así el orden se reduce a ley, a un principio: el principio de simplicidad, pues, no obstante que ve lo uno y a la vez lo múltiple, separa lo que está unido (disyunción) o re-úne lo que es disperso (reducción).

Recordemos que con los fundadores de la cibernética, Wiener y Ashby (en Morin, 2003), la noción de $<<$ complejidad $>>$ entra en escena en la ciencia; en tanto que con Von Neumann la complejidad se enlaza por vez primera a fenómenos de la auto-organización. Entonces, ¿qué entender por complejidad? Como lo expone Morin (2003), se puede distinguir esta como un fenómeno cuantitativo, "una cantidad extrema de interacciones e interferencias entre un número muy grande de unidades" (p.59). El mismo Morin agrega que un sistema viviente, auto-organizador, el más elemental, combina un gran número de unidades - del orden del billónpor ejemplo de moléculas en una célula, de estas en un organismo -más de 10 billones de células en el cerebro y más de 30 billones en el organismo humano-; empero la complejidad no radica solo en cantidades de unidades e interacciones que desafían al mismo cálculo, implica además incertidumbre, fenómenos aleatorios e indeterminaciones. Esto es, que la complejidad siempre se relaciona con el azar. Incluso, en el ámbito de la complejidad, se han establecido ciertos principios, a saber: a) Dialógico, que permite mantener la dualidad en el contexto de la unidad; agrupa nociones antagonistas y complementarias, b) Recursividad organizacional, como ocurre en el remolino que, es producido y a su vez es productor; y c) Hologramático, esto 
es, que la parte está en el todo como el todo en la parte.

En el pensamiento simplificante, según las apreciaciones de Morin (2003), hay un predominio de las operaciones lógicas: disyunción y reducción, ambas mutilantes mientras que el pensamiento complejo se basa por necesidad en: la conjunción, la distinción y la implicación.

De alguna manera, la aceptación de la complejidad es el reconocimiento de una contradicción, pero, en todo caso, no se pueden regatear las contradicciones del mundo. En su tiempo, Heráclito sostuvo: $<<$ Vivir de muerte, morir de vida $>>$ y también planteó que: hay armonía en la desarmonía, y al revés.

La complejidad en tanto propiedad sistémica ocurre en todos los dominios de la vida, trátese de los ámbitos biológico, cultural -el lenguaje, los símbolos- físico, social, político. Puede considerarse como una idea guía del pensamiento y acción que posibilita la comprensión y la comprensión de una realidad dinámica, en constante movimiento. Puede entenderse también, según Ciurana (2000), como un "concepto piloto" e, incluso, como hipótesis de trabajo inicial, con aspiración de un valor universal que suma a la lectura y comprensión de los fenómenos naturales y sociales, pues involucra diversos niveles de realidad.

La complejidad emerge en el sistema y en la organización, al pensar en la totalidad; se trata de la unitas multiplex de Morin, que reafirma Ciurana (2000):

La unidad en la diversidad que, simultáneamente, crea y reprime el antagonismo. Se trata de la unidad que posee una organización y función (visibles), pero también una parte oculta de antagonismos latentes, que es la que induce una potencialidad desorganizadora, desintegradora y, a su vez, creadora. (p.2)

Ahora bien, la idea de complejidad en el ámbito educativo va arraigándose paulatinamente como una estrategia metodológica junto a la transdisciplinariedad; y ambas configuran una clara postura epistemológica. Se vienen percibiendo como una guía para el pensamiento actual; de la complejidad que refiere a la forma de caracterizar al ser y la realidad; así como a la manera de organizar las vidas e ideas humanas. Comprende por tanto las dimensiones -o niveles dirá Nicolescu (1996)- de la naturaleza ontológica, epistémica y metodológica en la comprensión y operación -observa Moraesdel "paradigma educativo emergente, también reconocido como Eco-Sistémico". Tal planteamiento se despliega en la idea del Pensamento Eco-sistémico, avalado por la Organización de las Naciones Unidas para la Educación, la Ciencia y la Cultura (UNESCO), cuya autora es María Cándida Moraes (2013).

En este marco de argumentación, la transdisciplinariedad, que interpreta y propone Moraes, revalora y rescata la conciencia ecológica y 
relacional; articula la lógica del tercero incluido que dinámicamente involucran un sistema de percepción y de valores que implica religación de saberes; contribuye a la percepción de la complementariedad entre unidad y diferencia, la no contradicción, la incompletud de procesos. Tal sistema de percepción y conciencia conlleva a una ética ecológica, centrada en la diversidad y pautada en la solidaridad, la responsabilidad y la sustentabilidad. De ahí se desprende, además, una orientación educativa que contribuya en una materialización deseable respecto a la reforma del pensamiento y evolución de la conciencia; pues corresponde a la educación alentar la emergencia de una civilización de la $<<$ re-ligación $>>$, sintonizada con la sinergia de la revalorización de la vida con atención a la interdependencia de los procesos vitales, los ecosistemas, la Tierra toda. Lo anterior como posibilidad de superación de los impedimentos para "reflexionar sobre la complejidad", como bien analiza Santiago (2016, p.246).

\section{De la complejidad a la inter/transdiscipi- nariedad}

En la generación y recreación del conocimiento emerge y se pone a prueba la noción de complejidad; ello implica ineludiblemente pasar de los saberes disciplinares, como observa Santiago (2016, p.244) a la interdisciplinariedad. Y, de acuerdo con el proyecto de Edgar Morin:

La complejidad se impone de entrada como imposibilidad de simplificar; ella surge allí donde la unidad compleja produce sus emergencias, allí donde se pierden las distinciones y claridades en las identidades y causalidades, allí donde los desórdenes y las incertidumbres perturban los fenómenos... (...) allí donde las antinomias hacen divagar el curso del razonamiento. [...] La complejidad emerge como obscurecimiento, desorden, incertidumbre, antinomia. Esto mismo, que ha provocado la ruina de la física clásica, construye la complejidad de la physis nueva. (En García, 2008, p.19)

No obstante lo anterior, sostiene Rolando García, un epistemólogo que trabajó al lado de Jean Piaget, "no hubo tal ruina de la física clásica" y, más allá de "sus inaceptables extrapolaciones y generalizaciones, es indiscutible que Morin contribuyó a demoler las bases del racionalismo tradicional"; que dominaba, como en muchos otros espacios y culturas, el sistema educativo francés. García (2008) aporta una concepción de los sistemas complejos, entendidos como "una representación de un recorte de esa realidad, conceptualizado como una totalidad organizada (de ahí la denominación de sistema), en la cual los elementos no son "separables" y, por tanto, no pueden ser estudiados aisladamente" (p.21). En esta acepción de sistemas complejos se pone en relación el objeto de estudio y las disciplinas, desde las cuales se despliegan diversidad de estudios.

Las disciplinas son el resultado de visión en la que el conocimiento se ha ido clasificando por ciencias; así, en una acepción primigenia, disci- 
plina es entendida como "una ciencia separada de otras". Desde Platón y hasta el siglo XVIII, las ciencias se agruparon de acuerdo con las facultades del alma que actuaban predominantemente en cada una de ellas, recuerda García (2008), y precisa que Bacon, en la época de Galileo, aún defendía la vigencia de esta clasificación: ES una partición muy verdadera del conocimiento humano, aquella que se extrae de las facultades del alma racional, la cual es el asiento propio del conocimiento: la historia corresponde a la memoria, la poesía a la imaginación, la filosofia a la razón.

La distinción disciplinar continúa con Jeremy Bentham y André Ampére, quienes diferenciaron entre ciencias de la naturaleza y ciencias del hombre; este último también introduce la dicotomía entre "ciencias cosmológicas" y “ciencias noológicas" (del griego nous/noésis: mente, intelecto). Morin, por su parte, propuso el término "noosfera" para referirse al mundo de las ideas y la cultura. Wunt, a su vez distinguió entre ciencias de la naturaleza y "ciencias del espíritu”; y Windelband (en García, 2008) defendió la dicotomía entre ciencias "nomotéticas" e “ideográficas". En este devenir, la revolución científica del siglo XVII que representó Mathematical Principles of Natural Philosophy de Newton, fue rechazada por los cartesianos, que las consideraron como meras descripciones geométricas; pero fueron luego los franceses, en el transcurrir del siglo XVIII, quienes contribuyeron a completar y mostrar pruebas empíricas de la teoría. Rememora García (2008) que:
"Las "meras descripciones" de la mecánica de Newton, que ni siquiera eran admitidas como "Física", habían pasado a ser, en poco más de un siglo, el paradigma dominante de toda ciencia" (p.24). Posteriormente, a comienzos del siglo $\mathrm{XX}$, entran en crisis conceptos básicos de la ciencia como: causalidad, espacio, materia, tiempo; lo anterior debido a que "la concepción newtoniana de la Física había caducado", según observa García (2008, p.30). Esta crisis motivó a reconsiderar los fundamentos de la ciencia toda, y llevaron a un análisis de las cuestiones epistemológicas; dicha empresa la asumen escuelas neopositivistas de Viena y Berlín, que, observa García (2008), intentaron "una reformulación rigurosa de las posiciones empiristas que culminarían en el Empirismo Lógico. A partir de allí se inició el más ambicioso intento de unificación de todas las ciencias desde una perspectiva estrictamente reduccionista" (p.30). Una de las tesis principales ha sido, sin duda, la "unidad de la ciencia", cuyo representante es Rudolph Carnap quien, afirma: “...hay una unidad del lenguaje en la ciencia, es decir, una base común de reducción para los términos de todas las ramas de la ciencia, y esta base consiste en un estrecho y homogéneo conjunto de términos del lenguaje de objetos físicos (The physicalthing-language)" (Carnap en García, 2008, p.30).

En este ámbito, la noción de sistemas complejos, mismos que se conforman por elementos heterogéneos en interacción, implica que sus subsistemas forman parte de los "dominios materiales" de disciplinas diversas. 
En tanto que la interdisciplina supone una integración de distintos enfoques disciplinarios; lo cual exige que los individuos de todo equipo de investigación conozcan bien o sean expertos en su propia disciplina. Ahora bien, es posible abordar cualquier problema, propone García (2008), como "un sistema" donde sus elementos están interdefinidos $\mathrm{y}$, por tanto su estudio precisa de la coordinación de enfoques varios que deben ser integrados en uno común. De ahí que la interdisciplina implica "el estudio de problemáticas concebidas como sistemas complejos y que el estudio de sistemas complejos exija de la investigación interdisciplinaria” (p.37).

Encontramos entonces que la perspectiva en el pensar complejo es la inter y la transdisciplina, lo que significa ahora 'indisciplinaria', si se considera a la actual ciencia en tanto institución enorme y burocratizada que representa un todo de principios que resiste al mínimo cuestionamiento, al tiempo que excluye y violenta aquello que surge al margen de su modelo, tildándolo de 'no científico'.

La cuestión, sugiere García (2008), es: si el espíritu del hombre es insuficiente para aprehender el gran conjunto del saber disciplinario, es preciso, entonces, cambiar dicho espíritu o bien al saber disciplinario. Por su parte, Morin (2003) se propuso desarrollar, más allá del reduccionismo y del holismo, la noción de 'unidad compleja', que conecta al pensamiento analítico-reduccionista con el pensamiento global en una dialéctica. La lógica predominante de Occidente es de carácter homeostático, en el sentido de que guarda un equilibrio en los argumentos y discursos, expulsa la contradicción y el error, y controla y dirige los desarrollos del pensamiento.

De acuerdo con la Carta de la Transdisciplinariedad (Portugal, 1994), las tres características fundamentales de la actitud y visión transdisciplinaria son: rigor, apertura y tolerancia. Rigor en los argumentos, tomando en consideración todos los datos existentes como la mejor barrera frente a las posibles distorsiones. La apertura involucra la aceptación de lo desconocido, lo inesperado y lo imprevisible. La tolerancia, por último, implica el reconocimiento del derecho de ideas y verdades opuestas a las propias.

La transdisciplinariedad se puede caracterizar a su vez como: a) actitud: formación de un espíritu abierto a los enlaces y a lo desconocido; y b) estrategia: conjugación de distintos tipos de conocimientos (disciplinarios y extradisciplinarios), que permite -propone- la articulación de actores diversos para la producción de un conocimiento pertinente.

Ahora bien, lo que interesa y precisa atención es cómo, a partir de propuestas conceptuales -complejidad, transdisciplina-, se ha llegado a acciones y prácticas docentes y de investigación universitarias, tanto a nivel de agentes como de instituciones, en este caso de educación superior.

Allende las disciplinas se pueden ver las prácticas inter $\mathrm{y}$ transdisciplinarias como intentos inéditos de abordar objetos de estudio 
de naturaleza compleja por su multidimensión. Queda clara así la insuficiencia de una sola perspectiva disciplinar para estudiar e investigar hechos y fenómenos que escapan a toda visión reduccionista. Aquí se analiza el esfuerzo y trabajo transdisciplinario por establecer otras metodologías -y con ello epistemologías- ante nuevos objetos de estudio que involucran valores y lenguajes. La Universidad, en este contexto, es escenario, en la docencia e investigación, del autoanálisis y la re-estructuración en cuanto a caminos de indagación se refiere. Hay coincidencia de que la transdisciplina es $<<$ actitud $>>$ y $<<$ estrategia $>>$ ante problemáticas complejas que demandan no solo comprensión y explicación, sino también solución. Plantea nuevas metodologías que de alguna manera se vislumbran en programas y trabajos de posgrado.

En el contexto de las prácticas docentes y de investigación inter y transdisciplinarias, ha sido posible abordar problemas de diversa índole que, desde una sola óptica o perspectiva disciplinar, son insuficientes para precisar su diagnóstico, comprensión y explicación; como son los casos de la corrupción -no biológica, sino cultural, humana, como ethos enquistado en el ser social-, educación, salud, pobreza y la sustentabilidad. La instrucción escolar, la institución y el proceso educativo en tanto fenómenos humanos pueden abordarse en su comprensión e intervención, asumiendo su grado de complejidad, dados los diversos niveles que implica, como las fuerzas y energías económicas, políticas, intelectuales y de capacidades físicas. En este marco, ahora entra el tema de la sustentabilidad mirando a un futuro inmediato, a efecto de procurar preservar biodiversidades y recursos naturales que aseguren la vida.

Ahora se afirma que:

...la Educación para el Desarrollo Sostenible (EDS) exige métodos participativos de enseñanza y aprendizaje que motiven a los alumnos y les doten de autonomía, a fin de cambiar su conducta... (...) la EDS promueve la adquisición de competencias tales como el pensamiento crítico, la elaboración de hipótesis de cara al futuro y la adopción colectiva de decisiones. Exige cambios de gran calado en los métodos pedagógicos que se aplican actualmente. (UNESCO, 2005-2014)

Las cuestiones educativas como prioridades inmediatas deberán orientarse, en consecuencia, hacia los procesos de construcción del conocimiento y el aprendizaje; hacia la sustentabilidad ecológica, la ciudadanía planetaria como resultado de una $<<$ evolución del pensamiento $>>$, y hacia la inteligencia y la conciencia humanas desde una perspectiva holista o de integración. Deberán considerarse, además, la interculturalidad, la diversidad y el desarrollo pleno de la conciencia respecto a la sustentabilidad. La educación, en este sentido, es fundamental, pero habrá que ir más adelante de la simple coexistencia pacífica o pasiva; habrá que caminar, precisa Moraes (2013), hacia "una coexistencia activa, conscientemente actuante, ejercitando la participación, la solidaridad y la compasión. Esto porque sabemos que existe 
interpenetración sistémica en términos de energía, materia e información, entre lo uno y lo múltiple, entre lo simple y lo complejo, entre la unidad y la diversidad" (p.3).

En muchos espacios universitarios se trabaja en torno a la ecología de los saberes, los temas sobre complejidad y transdisciplinariedad cada vez más son comunes en la educación. Hay quien estima que, como educadores -quienes participamos en los procesos áulicos, de enseñanza y aprendizaje- debemos enfatizar en la correlación estrecha entre el tejido social, ecológico y planetario, que es común para todos; resaltar las interrelaciones constitutivas de naturaleza eco-sistémica que suceden en los distintos ámbitos naturales; entre otros, precisa Moraes (2013),

la interdependencia entre ambiente, ser humano, pensamiento y desarrollo. Necesitamos también acentuar la existencia de una cooperación global no solo en relación a los procesos cognitivos, emocionales e intelectuales, integrando razón, emoción, sentimiento, imaginación e intuición, sino también en lo que se refiere a las escalas social, ecológica y planetaria". (p.3)

Entre estas instancias ocurre una estrecha, compleja y vital correlación, más allá de la instrumentación y visión utilitaria que irresponsablemente se hace de los recursos que brindan los ecosistemas. Esta situación se está revalorando debido a las diversas crisis que impactan a la naturaleza y a la Tierra en su conjunto, así como a la vida toda. En tal escenario la educación tiene un rol importante que asumir.

La transdisciplinariedad es una posición teórica, metódica y epistemológica que plantea la existencia de otras realidades distintas a la que históricamente ha mostrado la ciencia y física modernas, y cuya visión-explicación de la naturaleza predomina desde la diversidad disciplinar. Nicolescu formado académica y profesionalmente en la física clásica, ha dado un paso más allá de ella arribando al universo cuántico. Su posición al respecto la ha expuesto en el Manifiesto sobre La transdisciplinariedad; ámbito de ideas en el que ha explorado la poesía como una posibilidad expresiva de sus asertos.

\section{Complejidad e interdisciplina}

La cuestión que sale a flote al examinar estos conceptos es la relación dualista: sujeto-objeto. El método de la racionalidad moderna, base de la ciencia contemporánea, que prescribe como norma del proceso de investigación el control, la delimitación (o fracción) del objeto para su análisis, experimentación y síntesis, es puesto en duda por el exceso de reducción y exigente control del <<error $>>$ que, en el paradigma de la complejidad, se mira como parte del proceso de conocer. Si bien el conocimiento arroja luz en la comprensión de los hechos y fenómenos, genera al mismo tiempo sombras -partes oscuras (errores)-, que no se deben desestimar, pues son consecuencia de aquello, comenta metafóricamente Morin (2003).

Por eso Panikkar (2006) previene respecto a 
caer en la trampa de separar las partes del todo, dado que es un ardid

del estudio de un ente 'en sí mismo' (objetividad) separado del 'yo mismo' (subjetividad) y también del 'Mismo' (trascendencia inmanente). Admitido que la ciencia moderna sea una magnífica conquista de la mente humana, sería totalitarismo cultural identificarla con la cultura, considerándola como la única forma de pensamiento propia del hombre". (p.584)

Precisa Pannikar (2006) que no es la ciencia pura quien afirma esto, sino la civilización tecnocientífica que envuelve nuestras vidas hoy, y que no oferta 'ningún otro modelo' para concebir el mundo; por lo que hoy en día se aplica al todo 'lo que caracteriza solo a una parte'. Es obvio entonces el peso que tiene hoy día la visión 'científica' del mundo; sin embargo también resulta extremadamente reduccionista, carente de imaginación y, por tanto, monocultural. El mismo Panikkar (2006) afirma que la tesis es especiosa, dado que la cultura científica parte del supuesto de que el lenguaje matemático es fiel reflejo de la realidad; no obstante, aunque esta proposición tiene su lógica (él dice: 'su fuerza') resulta harto simplista, pues, si bien la naturaleza está descrita matemáticamente, cabe observar que "la escritura no es la realidad", como un mapa no es el territorio.

Entonces tenemos que el modelo de pensamiento científico tan solo es un paradigma-muy específico- y como tal, aún siendo magnífico en su propio contexto, provoca el deterioro del universo de símbolos de otras culturas cuando se extrapola. Concluye Panikkar (2006) que, "el poder de la visión científica del mundo es obvio pero esta es excesivamente reduccionista, monocultural y carente de imaginación, como si el homo tecnologicus fuese sinónimo de Homo sapiens" (p.585).

Volviendo a la idea según la cual la naturaleza está escrita en lenguaje matemático, restaría leerla y posteriormente interpretarla. En tal sentido, la ciencia moderna a través de sus actores asume la creencia en la posesión de una clave interpretativa, trazada en postulados matemáticos. No obstante, es posible mostrar la existencia de otras claves; dicho de otra forma, "que el libro de la naturaleza es susceptible de muchas interpretaciones y que la ciencia es solo una de ellas" (Panikkar, 2006). Tal es el caso de la actual encrucijada histórica, desafío verdadero de la globalización.

El dilema es inevitable-Sostiene Panikkar (2006): o la civilización tecno-científica, con su concepción del hombre como individuo, de la materia como simple masa, de la verdad como verificable (o falseable) y de la realidad como dato empírico, es superior a cualquier otra cultura de la que se integran los aspectos $<<$ positivos $>>$ por medios pacíficos, realizando el paso, respetando los ritmos históricos y agilizando el dinamismo de la historia sin falsos sentimentalismos, o existen también otras culturas que permiten igualmente al hombre alcanzar su plenitud y realización y su felicidad". (p.585) 
Admitir que la $<<$ cultura científica $>>$ es superior a todas las demás, es caer una vez más en un exceso de reduccionismo.

Esa ciencia que está en pos de la certeza se afianza a ella, precisamente, por la aparente confianza que brinda. La búsqueda de la certeza se remonta al temor a la vida y a la muerte -siempre inciertas con relación al tiempo- y en la idolatría a un tipo de razón que ofrece dicha certeza: que la certidumbre se basa en la evidencia racional que se desvela en la propia razón. Pero, en realidad, mediante ella emerge un círculo vicioso. "Es la misma razón la que nos dice que podemos fiarnos de ella, una vez que haya alcanzado la claridad de la evidencia. La certeza es la confianza de la razón en sí misma que ella misma avala" (Pannikar, 2006, p.586). En oposición a la certeza aparece la confianza, que surge

cuando nos damos cuenta de que nuestra propia naturaleza nos empuja a confiar en algo que, aun no siendo nosotros mismos, está en nosotros, de que no estamos solos sino ligados al todo, cosa que lleva a la confianza cósmica, que parece ser el impulso más profundo de lo creado y nuestra tendencia más natural. (Pammikar, 2006, p.586)

Confianza denota fe, fiarse en algo o alguien; y nos fiamos porque creemos, esto es

porque el corazón nos empuja a hacerlo y la razón no pone un veto: una vez más no es posible la separación entre conocimiento y amor sin que ambos degeneren. La confianza se basa en la fidelidad de las cosas, es decir, en su auto-identidad. La confianza es más profunda que la certeza, que se basa solamente en la confianza en la razón, aunque el papel de esta en cualquier caso sea indispensable. (Panikkar, 2006, p.587)

Para contextualizar el pensamiento de este autor cabe decir que abreva de la mística hindú por ascendencia familiar y de la filosofía y fe cristiana como catalán, por nacimiento.

En el pensamiento occidental se entronizó a la razón por encima de todas las culturas humanas; Las ciencias comparadas aparecen "desde el momento en que se creía haber encontrado en la Razón la instancia suprema que permitía compararlas e incluso juzgarlas" (Panikkar, 2006, p.587). Y en ese contexto cada quien, en su momento, defendió la propia razón, pero la "fe" en el mito de la razón comienza a resquebrajarse; no obstante las interesantes distinciones que la filosofía contemporánea realiza en torno a los diferentes tipos de Razón: pura, práctica, instrumental, histórica, comunicativa.

Al planteamiento o creencia entre mitológica y científica según la cual "todo está ligado a todo", que podría traducirse como una respuesta a tanta fragmentación de la vida, que ha conducido a la segmentación del conocedor, Panikkar (2006) resalta al respecto el divorcio entre conocimiento y amor que, usualmente, son estimadas como facultades independientes del ser humano. Sostiene que "conocimiento sin amor es mero cálculo, no penetra en lo que conoce; amor sin conocimiento es simple emoción, no hay identificación con lo que se ama" (Pannikar, 2006, p.587) Y ejemplifica: 
La Ilustración europea representa el esfuerzo genial de basar la vida humana individual y la política en la racionalidad: el hombre es un animal racional y la razón es el criterio último de verdad $\mathrm{y}$, por tanto, de realidad. Desde Descartes a Husserl se desarrolla la espina dorsal de la modernidad y de todas sus creaciones, desde la "ciencia" a la "democracia". Los principios, tanto del pensamiento como de la acción, han de ser principios racionales. Kant podría ser el paradigma de ello. La razón es la reina, su reino es por definición la inmanencia: la inmanencia es todo aquello que cae dentro del campo de la razón. Si hay algo "fuera" es irracional, no humano; (...) y el conocimiento no puede sobrepasar los fenómenos, es decir, aquello que cae dentro del campo de la razón. (...) La razón debe ser el fundamento de todo; pero ¿cómo puede la razón “dar la razón” de sí misma? Schelling luchará desesperadamente para encontrar un fundamento al Yo absoluto y escapar del monismo de Hegel. La intuición estética es su respuesta $-y$ aquí comienza la crisis de la modernidad: un arte que escapa a los cánones de la racionalidad. (Panikkar, 2006, p.140)

Ahora bien, conocer todas las cosas deriva del conocerse a sí mismo; y a su vez, "conocer al otro es conocerse a sí mismo porque nuestro yo no es una mónada aislada y el otro, como alter, es "parte" del yo. Así que "conocerse a sí mismo es conocer a todas las cosas", así afirman Proclo, IbnSina, Meister Eckhart, entre muchos otros. En este marco, precisa Panikkar (2006), se debe tener presente que, "la realidad no está fragmentada, cercenada en partes sino que, subsiste en su propia a-dualidad, que es la de una conectividad radical" (p,585).

\section{Resultados epistémicos}

Como consecuencia de novedosas lecturas e interpretaciones de la realidad a partir sobre todo de la física contemporánea, y particularmente de la mécánica cuántica, que pone en entredicho las leyes de la física nuclear en cuyo paradigma aún nos desenvolvemos, no obstante la presencia discursiva y experimental de la física cuántica desde mediados del siglo XX; el reduccionismo y causística de la ciencia moderna, cuantitativa y experimental, aún prevalece. A ello se antepone la posibilidad de una interpretación y comprensión de lo Real, desde la perspectiva holista y sistémica que aborda y mira procesos -complejos- como totalidades. La discusión radica en la pretension del conocimiento que se aspira a generar; este regularmente ha sido la parte o fracción de un todo desde donde se ha deshilvanado un saber, como ocurre en las teorías molecular, celular o de la evolución (de la vida), que, a falta de explicaciones mejores, constituyen todo un paradigma de comprensión. La complejidad como propuesta metodológica, epistémica y teórica resulta una alternativa ante la arrogancia de esa racionalidad científica, instrumental y tecnológica. $\mathrm{Y}$ con ella la práctica transdisciplinaria como opción metodológica en el abordaje de problemas de diversa índole, que, en sí mismos, por su constitución e interrelación material y vital, son de naturaleza compleja. Todo ello apunta a que lo que subyace 
en el fondo es un problema epistemológico que también puede ser abordado desde la complejidad y la transdisciplinariedad; muestra de ello es que en las prácticas docentes y de investigación universitarias resultan un hecho de sumo ordinario a través de diversas asignaturas y programas de grado y posgrado. Lo anterior se pone de manifiesto, por otro lado, con el tema de la sustentabilidad que, dadas las dimensiones que involucra, resulta insuficiente para una sola disciplina científica, como ha ocurrido, no obstante, con la obstinación por la economía y la política, perspectivas predominantes pero ideológicamente cuestionadas.

\section{Discusión y conclusiones}

Como hemos afirmado en otro momento (Salvador, 2012a), la manera en que los seres humanos habitamos el mundo en este siglo XXI, aun en remotos sitios, está permeada por la tecnología, para bien o para mal; si bien el humano es natural $\mathrm{y}$, por ende, no puede renegar de su basamento terrenal, también es cierto que que ha creado para sí el artificio de un segundo hogar más allá del natural: la comunidad-ciudad y con ella, simultáneamente, el lenguaje y la cultura. De hecho, tenemos en la propia tecnología un problema más del orden complejo y transdisciplinar; siendo material en cuanto a su composición de partes y artefactos, pero, son la autonomía, la libertad y voluntad humanas (personales), quienes le dan sentido y pertinencia.

Como el conocimiento reconoce la multidimensionalidad del fenómeno y, por ende, la insuficiencia de un abordaje parcial y disciplinar; son pertinentes las perspectivas compleja y trans- disciplinar, no solo de manera metodológica, sino teórica y epistemológicamente. Recordemos que, antes de la ciencia moderna, fue el saber filosófico, desde la reflexión en torno al cosmos, la naturaleza y el propio hombre, el que generó un saber de alcance universal (Salvador, 2012b). Ahora, el pensamiento filosófico y moral asumen un rol protagónico en el análisis del conocimiento científico, que inolucra la naturaleza, los recursos y sus ecosistemas, la biosfera, entendida como los elementos que le confieren viabilidad y sentido a la existencia: el agua, la tierra y el aire, sin los cuales no hay proyecto vital. Ante las severas crisis mundiales que ponen en inminente riesgo no solo la vida, sino la propia Tierra como único hábitat natural para el ser humano y el resto de seres vivos, no queda sino la comprensión desde una complejidad sistémica, holística y/o mística si se quiere. Recordemos al respecto que la teoría de Gaia interpreta a la Tierra como un ser vivo que se autorregula, y en muchas partes del mundo hay movimientos, de pensamiento y acciones correpondientes, que reivindican a la naturaleza y la vida. Todo ello es posible de interpretar y abordar desde la perspectiva transdisciplinar, entendiéndola en tanto un arte y una ciencia del descubrimiento, un diálogo de saberes diversos, heterogéneos, contradictorios, acaso. La transdisciplina radica de hecho en la educación, preferentemente en la superior. Reconozcamos que como posibilidad puede brindar muchos y buenos resultados, todo depende de la superación de los reductos disciplinares que nos atan y limitan a interpretaciones reduccionistas $\mathrm{y}$ parciales de las disciplinas en las que fuimos formados $\mathrm{y}$, consecuentemente, nos sentimos 
cómodos. Dar un paso más allá, como lo sugiere el saber transdisciplinar, demanda compromiso y rigor; no obstante, como lo hemos sostenido (2012b), se debe tener cuidado para evitar que pronto se convierta en $<<$ programa de acción $>>$ científico e ideológico, lo que podría acarrear consecuencias indeseables: siempre será preferible la duda y el cuestionamiento a la unanimidad ciega en una razón, desplegada paradójicamente desde la emoción.

\section{Referencias}

Capra, F. (2007). Sabiduría insólita. Conversaciones con personajes notables. (3a. ed.) Barcelona: Kairós.

Ciurana, E.R. (2000). El modelo organizacional, su método. En: http://www.iecomplex. com.br/textos/elmolde.htm (A c c e s o 14/09/13).

Francois, C. (2006) "El mundo real es una complejidad organizada que demanda una visión sistémica”. En Megatendencias. Disponible en http://www.tendencias21. net/El-mundo-real-es-una-complejidadorganizada-que-demanda... (Consulta: 28 de marzo 2011)

Flórez M. C. (2001). Introducción. Descartes. Barcelona: Gredos.

García, R. (2008). Sistemas complejos. Conceptos, método y fundamentación epistemológica de la investigación interdisciplinaria. Barcelona: Gedisa.

Moraes, M. C. (2013). Complejidad, Transdisciplinariedad y Educación: algunas reflexiones. Brasil: PUC/SP (Traducción: Pedro García González). http://www. encuentros-multidisciplinares.org/
Revistan \% C 2\% B A $25 / \mathrm{Mar} \% \mathrm{C} 3$ $\%$ ADa\%20C\%C3\%A1ndida\%20Moraes. pdf

Morin, E. (2003). Introducción al pensamiento complejo ( $6^{\mathrm{a}}$ reimp). Barcelona: Gedisa.

Nicolescu, B. (1996). La transdisciplinariedad. Manifiesto. México: Multiversidad Mundo Real "Edgar Morin", A.C.

Panikkar, P. (2006). Paz e Interculturalidad. Una reflexión filosófica. Barcelona: Herder.

Reale, D. \& Antiseri D. (2001). Historia del pensamiento filosófico y científico. (4a. ed.). Barcelona: Herder. http://www.rsu. uninter.edu.mx/doc/glosario/Transdisciplinariedad.pdf

Salvador B., J.L. (2012a). Ethos y complejidad: hombre, naturaleza y vida. ¿Hay lugar para la persona hoy? El problema de la ubicación de la persona humana en nuestro tiempo (pp.77-107). Rosario: UCEL.

Salvador B., J.L. (2012b). Complejidad, ciencia y sociedad. Ethos del conocimiento simple versus complejo. Great Britain: Amazon.

Santiago, J. A. (2016). La acción de las Ciencias Sociales y el desarrollo del pensamiento crítico. Revista Educación y Humanismo, 18(31), 241-456. http:// dx.doi.org/10.17081/eduhum.18.311377

UNESCO (2005). Decenio de las Naciones Unidas de la Educación para el Desarrollo Sostenible 2005-2014. El Decenio en pocas palabras. http://unesdoc.unesco. org/images/0014/001416/141629s.pdf 